\title{
Realtime Monitoring of Vascular Conditions Using a Probabilistic Neural Network
}

\author{
Akira Sakane $^{1}$, Toshio Tsuji ${ }^{1}$, Yoshiyuki Tanaka ${ }^{1}$, \\ Kenji Shiba ${ }^{1}$, Noboru Saeki ${ }^{2}$, and Masashi Kawamoto ${ }^{2}$ \\ 1 Department of Artificial Complex Systems Engineering \\ Hiroshima University \\ Kagamiyama 1-4-1, Higashi-Hiroshima, Hiroshima, 739-8527 JAPAN \\ \{sakane, tsuji, ytanaka, shiba\}@bsys.hiroshima-u.ac.jp \\ http://www.bsys.hiroshima-u.ac.jp \\ 2 Department of Anesthesiology and Critical Care \\ Hiroshima University \\ Kasumi 1-2-3, Minami-ku, Hiroshima, 734-8551 JAPAN \\ \{nsaeki, anekawa\}@hiroshima-u.ac.jp
}

\begin{abstract}
This paper proposes a new method to discriminate the vascular conditions from biological signals by using a probabilistic neural network, and develops the diagnosis support system to judge the patient's conditions on-line. For extracting vascular features including biological signals, we model the dynamic characteristics of an arterial wall by using mechanical impedance and estimate the impedance parameters "beat-to-beat". As a result, this system can be utilized for the actual surgical operation, and the vascular conditions can be discriminated with high accuracy using the proposed method.
\end{abstract}

\section{Introduction}

A physician needs to judge patient's conditions accurately and take appropriate measures. Especially, blood pressure and electrocardiogram are used to judge the patient's conditions during surgical operations, and the waveforms of these biological signals change based on the vascular conditions. However, due to the inevitability of the human error factor in combination with inexperienced physicians, the judge of a patient's condition will most likely not be 100 percent accurate. Therefore, medical diagnosis computer assisted technology on vascular conditions is helpful for physicians in supporting complex diagnosis. As the development of a diagnosis support system based on vascular conditions, the estimation system of health condition and vascular aging have been reported 1], [2]. For example, Maniwa et al. estimated a acceleration plethysmogram and judged the conditions of health or illness quantitatively using a recurrence plot which is one of the chaos approach [1. However, the proposed method does not correspond to long term-series behavior, and it is not applied to monitor the patient's conditions during operations. 


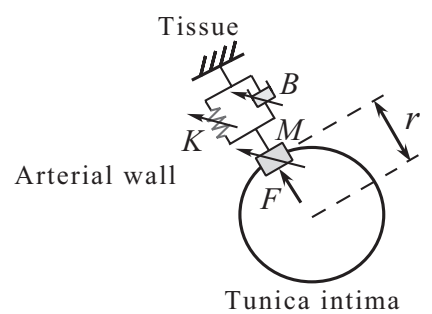

Fig. 1. An arterial wall impedance model

This paper aims to develop a diagnosis support system using a probabilistic neural network. In the many proposed techniques, we used Log-Linearized Gaussian Mixture Network (LLGMN) 3], based on the Gaussian mixture model (GMM) and the log-linear model of the probability density function. Specifically, about pattern discrimination of EMG signals, high discrimination capability is shown as compared with other neural networks 44. We have discriminated vascular conditions from measured biological signals by using the neural network, thus the vascular conditions could be discriminated with high accuracy [5], 6]. However, the data used for discrimination were measured in advance, and the availability was not evaluated in actual surgical operation.

This paper proposes a method to discriminate vascular conditions using a probabilistic neural network on-line, and the availability of realtime monitoring of vascular conditions is verified.

\section{Dynamic Characteristics of Arterial Wall}

For extracting vascular features including biological signals, we estimated the arterial wall impedance [5]. Fig. [1]illustrates the proposed impedance model of the arterial wall. This model represents only the characteristics of the arterial wall in the arbitrary radius direction. The impedance characteristic can be described using an external force and a displacement of the arterial wall as follows:

$$
d F(t)=M d \ddot{r}(t)+B d \dot{r}(t)+K d r(t)
$$

where $F(t)$ is the force exerted on the arterial wall by blood flow; $M, B$, and $K$ are the inertia, viscosity, and stiffness; $r(t), \dot{r}(t)$, and $\ddot{r}(t)$ are the position, velocity, and acceleration of the wall; the coefficient $d$ means the variation from the time $t_{0}$; and $t_{0}$ denotes the start time just at moving arterial wall.

To estimate the impedance parameters given in (1), it is necessary to measure $F(t)$ and $r(t)$. Assuming that the force $F(t)$ is proportional to arterial pressure $P_{b}(t)$, the following equation can be obtained:

$$
F(t)=k_{f} P_{b}(t)
$$

where $k_{f}$ is a proportional constant [5]. 
On the other hand, the vascular radius $r_{v}(t)$ is quite difficult to measure directly. So a plethysmogram is utilized instead of $r_{v}(t)$ as follows [5]:

$$
r_{v}(t)=\frac{P_{l}(t)+A_{D}}{k_{p}}
$$

where $P_{l}(t)$ is a plethysmogram, $k_{p}$ is a proportional constant, and $A_{D}$ is the absorbance.

The force exerted on the arterial wall is expressed by the arterial pressure $P_{b}(t)$ given by $(2)$, and the vascular radius $r_{v}(t)$ is represented by the plethysmogram $P_{l}(t)$ in $(3)$. Then, the arterial wall impedance is estimated by using $P_{b}(t)$ and $P_{l}(t)$ as follows:

$$
d P_{b}(t)=\tilde{M} d \ddot{P}_{l}(t)+\tilde{B} d \dot{P}_{l}(t)+\tilde{K} d P_{l}(t)
$$

where the parameter $\tilde{M}$ corresponds to the mass of the arterial wall existing in the measured part; $\tilde{B}$ and $\tilde{K}$ to the viscoelastic properties, respectively [5]. In the proposed method, the estimation period of the arterial wall impedance is the time between successive R-peaks (an RR interval), where the peak of the $\mathrm{R}$ wave can be detected from ECG signals. The impedance parameters $\tilde{M}, \tilde{B}$, and $\tilde{K}$ are estimated by substituting $d P_{b}(t)$ and $d P_{l}(t)$ into $(4)$, where $d P_{b}(t)$ and $d P_{l}(t)$ are the variations of arterial pressure and plethysmogram from the detecting time of $\mathrm{R}$ wave $t_{0}$. Because the time needed for estimating arterial wall impedance is smaller than the RR interval, it is possible to estimate the arterial wall impedance "beat-to-beat" [5].

We proposed the impedance ratio to reduce the individual differential [5]. The impedance ratios $\tilde{M}_{\text {ratio }}, \tilde{B}_{\text {ratio }}$, and $\tilde{K}_{\text {ratio }}$ are calculated for $\tilde{M}, \tilde{B}$, and $\tilde{K}$ as follows:

$$
\tilde{M}_{\text {ratio }}=\frac{\tilde{M}}{\tilde{M}_{\text {rest }}}, \tilde{B}_{\text {ratio }}=\frac{\tilde{B}}{\tilde{B}_{\text {rest }}}, \tilde{K}_{\text {ratio }}=\frac{\tilde{K}}{\tilde{K}_{\text {rest }}}
$$

where $\tilde{M}_{\text {rest }}, \tilde{B}_{\text {rest }}$, and $\tilde{K}_{\text {rest }}$ are the nominal values of impedance ratios when patients are in a relatively rested condition. Similarly, the ratios of arterial pressure and plethysmogram are calculated as follows:

$$
I B P_{\text {ratio }}=\frac{I B P_{\max }-I B P_{\min }}{I B P_{\text {rest }}}, P L S_{\text {ratio }}=\frac{P L S_{\max }-P L S_{\min }}{P L S_{\text {rest }}}
$$

where $I B P_{\max }, I B P_{\min }, P L S_{\max }$, and $P L S_{\min }$ are maximum and minimum values of arterial pressure and plethysmogram divided into beat-to-beat; $I B P_{\text {rest }}$ and $P L S_{\text {rest }}$ are maximum and minimum value differences of arterial pressure and plethysmogram in a relatively rested condition [5].

A neural network is used to discriminate the vascular conditions using the normalized ratios of impedance and biological signals given by

$$
\tilde{M}_{\text {ratio }}^{\prime}=\frac{\tilde{M}_{\text {ratio }}}{k_{\mathrm{M}}}, \tilde{B}_{\text {ratio }}^{\prime}=\frac{\tilde{B}_{\text {ratio }}}{k_{\mathrm{B}}}, \tilde{K}_{\text {ratio }}^{\prime}=\frac{\tilde{K}_{\text {ratio }}}{k_{\mathrm{K}}}
$$




$$
I B P_{\text {ratio }}^{\prime}=\frac{I B P_{\text {ratio }}}{k_{\mathrm{I}}}, P L S_{\text {ratio }}^{\prime}=\frac{P L S_{\text {ratio }}}{k_{\mathrm{P}}}
$$

where the gains for normalization, $k_{\mathrm{M}}, k_{\mathrm{B}}, k_{\mathrm{K}}, k_{\mathrm{I}}$, and $k_{\mathrm{P}}$ are determined by the maximum value of each estimated signal in advance [6].

The LLGMN receives the ratios of impedance and biological signals of (7).

\section{Monitoring Experiment}

\subsection{Experimental Condition}

The proposed method is applied to discriminate the vascular conditions. The subject was operated on using the endoscopic transthoracic sympathectomy for hyperhidrosis (Patient A : male 14 years). If a blood vessel contracts due to stimulation from sympathetic nerves, the palms and under arms will perspire. In this operation, the sympathetic nerves on the sides of the backbone are interrupted using a clip to stop the perspiration [7]. When the sympathetic nerve is interrupted, blood vessels become compliant on the spot. Therefore, if the vascular conditions can be identified on-line, it is possible to ascertain its success or failure during operation. Electrocardiogram $(E C G(t))$, arterial pressure $\left(P_{b}(t)\right)$, and plethysmogram $\left(P_{l}(t)\right)$ were measured at $125[\mathrm{~Hz}]$ simultaneously for discriminating vascular conditions. The arterial pressure was measured through a catheter (24 gauge) placed in the left radial artery, and the plethysmogram was measured with the ipsilateral thumb (BSS-9800, NIHON KOHDEN Corp.).

We constructed the diagnosis support system equipped with a graphical display screen using LabVIEW (National Instruments Corp.) in order for a physician to ascertain the patient's conditions easily. This system was tested to determine whether realtime monitoring of vascular conditions was possible.

The learning data was created from that of the four patients (nonsubjects) who operated the endoscopic transthoracic sympathectomy for hyperhidrosis patients. However, the learning data of the "Vasodilation" and "Shock" conditions were not available from the patients. Such unobservable facts were represented randomly by normal distribution with $N(\mu, 0.005)$. In this paper, means $\mu$ was set as follows: $\mu_{I B P_{\text {ratio }}^{\prime}}=0.12, \mu_{P L S_{\text {ratio }}^{\prime}}=$ $0.75, \mu_{\tilde{M}^{\prime}{ }_{\text {ratio }}}=0.006, \mu_{\tilde{B}^{\prime} \text { ratio }}=0.006, \mu_{\tilde{K}^{\prime}{ }_{\text {ratio }}}=0.006$ for vasodilation;

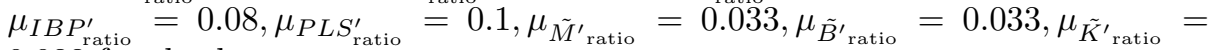
0.033 for shock.

\subsection{Experimental Result}

Fig.2 shows the result of discrimination experiment. Time profiles of the ratio of arterial pressure, the ratio of plethysmogram, the ratio of inertia, the ratio of viscosity, the ratio of stiffness, the shock index (SI), the coefficient of determination, and the classification results are shown in order from the top. In this paper, we defined the four vascular conditions i.e., I) shock, II) normal, III) vasoconstriction, and IV) vasodilation [5]. The coefficient of determination is 


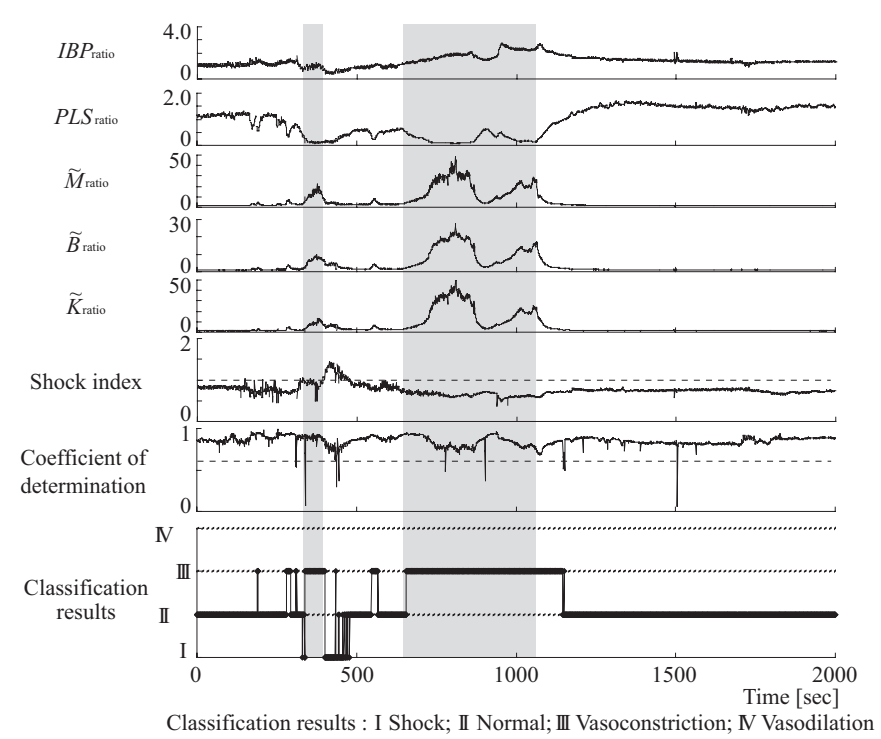

Fig. 2. Classification result of the vascular conditions during surgical operation

calculated from the measured arterial pressure and estimated arterial pressure using (4), and is used for judging whether discrimination should take place. Also, the shock index is used routinely for defining the "Shock" condition. This index is calculated from the ratio of heart rate to systolic arterial pressure; "Normal" condition at 0.6 ; while "Shock" condition over 1.0 [8].

The blood vessels become stiff in the shaded areas. The nominal values of impedance and biological signals are an average of consecutive 10 sample data after the patients are under anesthesia. The discrimination result is selected as vascular condition if the coefficient of determination $R^{2} \geq 0.6$. On the other hand, the discrimination is suspended if $R^{2}<0.6$. Also, the normalization gains included in (7) are set as follows: $k_{\mathrm{M}}=k_{\mathrm{B}}=k_{\mathrm{K}}=30.0, k_{\mathrm{I}}=2.5, k_{\mathrm{P}}=2.0$.

In Fig[2, the estimated impedance shows that the blood vessels gradually became stiff because the doctor stimulated the patient's tissues to find the sympathetic nerves at 300-400 and 700-1100 [sec], and NN discriminated the vascular condition as "Vasoconstriction". After 400 [sec], the ratios of arterial pressure decreased rapidly, the vascular condition was discriminated as "Shock", and the corresponding shock index was indicated as equal or greater than 1.0 at the same time. Also, the sympathetic nerves are distributed on the two sides of body, the nerve that was concerned the left arm's blood vessels was interrupted at 1100 [sec]. Therefore, after 1100 [sec], the blood vessels became compliant, and vascular condition was discriminated as "Normal". After that, the operation that was interrupted the sympathetic nerve of the other side of the left arm measuring the biological signals was occurred at 1200-2000 [sec]. Then, the left arm's blood vessels were not constricted if the sympathetic nerve was interrupted properly. The discrimination result indicated "Normal" condition, and it meant that the sym- 
pathetic nerve was interrupted well. From this result, it was confirmed that the vascular conditions could be monitored in realtime during operation and judged more easily. However, the coefficient of determination showed low value because the phase difference between blood pressure and plethysmogram was large. It is considered to be easily solved by improving the model in contemplation of the pulse wave velocity.

\section{Conclusion}

This paper proposed a new method to discriminate the vascular conditions by using a probabilistic neural network, and developed the diagnosis support system to judge the patient's conditions on-line. This system could be utilized for the actual surgical operation, and the vascular conditions could be discriminated with high accuracy using the proposed method. Future research will test the method's validity using different surgery.

Acknowledgment. The authors would like to thank T.Ukawa and K.Tone of the NIHON KOHDEN Corp. for making available clinical data and for their precious suggestions. This work was supported by Grant-in-Aid for Scientific Research of Japan Society for the Promotion of Science (15008279).

\section{References}

1. Maniwa, Y., Iokibe, T., Koyama, M., Yamamoto, M., Ohta, S.: The Application of Pulse Wave Chaos in Clinical Medicine, in Proc. 17th FUZY System Symposium, Chiba (2001) 787-790

2. Takada, H., Mirbod, S.M., Iwata, H.: The relative vascular age derived from acceleration plethysmogram: A new attempt., Jpn. J. Appl. Physiol., Vol. 28, No. 2 (1998) 115-121

3. Tsuji, T., Fukuda, O., Ichinobe, H., Kaneko, M.: A Log-Linearized Gassian Mixture Network and Its Application to EEG Pattern Classification, IEEE Trans. Syst., Man, Cybern-Part C, Appl. and Rev., Vol. 29, No. 1 (1999) 60-72

4. Fukuda, O., Tsuji, T., Kaneko, M. Otsuka, A.: A Human-Assisting Manipulator Teleoperated by EMG Signals and Arm Motions, IEEE Trans. Robotics and Automation, Vol.19, No.2 (2003) 210-222

5. Sakane, A., Tsuji, T., Saeki, N., Kawamoto, M.: Discrimination of Vascular Conditions Using a Probabilistic Neural Network, Journal of Robotics and Mechatronics, Vol. 16, No. 2 (2004) 138-145

6. Sakane, A., Tsuji, T., Tanaka, Y., Saeki, N., Kawamoto, M.: DEVELOPMENT OF A DIAGNOSIS SUPPORT SYSTEM ON VASCULAR CONDITIONS USING A PROBABILISTIC NEURAL NETWORK, in Proc. 2nd International Symposium on Measurement, Analysis and Modeling of Human Functions (2004) (in press)

7. Drott, C., Gothberg, G., Claes, G.: Endoscopic transthoracic sympathectomy: An efficient and safe method for the treatment of hyperhidrosis, Journal of the American Academy of Dermatology, Vol. 33 (1995) 78-81

8. Rady, M.Y., Nightingale, P., Little, R.A., Edwards, J.D.: Shock index: a reevaluation in acute circulatory failure, Resuscitation, Vol. 23, No. 3 (1992) 227-234 